\title{
Anterior uveitis associated with intravenous cidofovir use in patients with cytomegalovirus retinitis
}

\author{
Jayakrishna Ambati, Kevin B Wynne, Mary C Angerame, Michael R Robinson
}

\begin{abstract}
Aim-Intravenous cidofovir is used to treat cytomegalovirus (CMV) retinitis, and has been reported to cause anterior uveitis. Relations were sought between this complication and patient characteristics that might help predict its occurrence.

Methods-17 patients with AIDS and CMV retinitis who were treated with intravenous cidofovir were identified, and the following data collected in a retrospective chart review: demographic characteristics, duration of CMV retinitis, retinal lesion characteristics, dose and duration of cidofovir therapy, tests of renal function, CD4+ T lymphocyte counts, visual acuity, intraocular pressure, iris colour, history of diabetes mellitus, and use of concomitant medications. Case-control analyses were performed to determine risk factors for developing cidofovir associated uveitis.
\end{abstract}

Results-Anterior uveitis characterised by pain, ciliary injection, and decreased visual acuity occurred in 10 patients (59\%). Median interval to development of uveitis was 11 doses of cidofovir. Symptoms developed 4.4 (SD 2.5) days (median 3.5) after an infusion of cidofovir. Patients who developed uveitis had a significantly greater rise in CD4+ $T$ lymphocyte count while receiving cidofovir $\left(68.4(75.7) \times 10^{6} / 1\right.$ versus $\left.5.0(0.6) \times 10^{6} / 1,(p=0.04)\right)$. By stepwise linear regression, this factor accounted for $33 \%(p=0.03)$ of the effect of developing uveitis. Mean follow up time, intraocular pressure decline during cidofovir therapy, serum creatinine and urine protein concentrations, and rates of protease inhibitor use were not significantly different between patients who developed uveitis and those who did not. Uveitis responded to topical corticosteroids and cycloplegia.

Conclusion-Anterior uveitis in patients receiving intravenous cidofovir therapy may be related to improving immune function. The uveitis responds to treatment and may not preclude continuation of cidofovir.

(Br f Ophthalmol 1999;83:1153-1158)

Michael R Robinson, MD, 10 Center Drive, National

Eye Institute/National

Institutes of Health, Building

10 , Room 10N112,

Bethesda, MD 20892, USA.

Accepted for publication 28 May 1999
Cidofovir ([S]-1-[3-hydroxy-2-phosp methoxypropyl] cytosine, Vistide, Gilead Sciences, Foster City, CA, USA), an acyclic nucleotide analogue, is effective in delaying progression of cytomegalovirus (CMV) retinitis. $^{1-5}$ Non-granulomatous uveitis has been reported to be a complication in $26-44 \%$ of patients receiving intravenous cidofovir ${ }^{6}$ and $24 \%$ of patients receiving intravitreal cidofovir under an investigational protocol. ${ }^{8}$ The cause of this reaction is unknown but has been associated with protease inhibitor use, ${ }^{6}$ suggesting that patients with better immune function are at increased risk, and possibly explaining why recently reported rates of uveitis are higher than those reported during the drug's development. In patients receiving intravitreal injections of cidofovir the rate of anterior uveitis appears to lower with concomitant use of probenecid through unknown mechanisms. ${ }^{8}$ We studied all patients receiving cidofovir for CMV retinitis at our institution to gain additional insight into factors related to the development of drug associated uveitis.

Materials and methods

We conducted a retrospective chart review of all patients with AIDS and CMV retinitis who were treated with intravenous cidofovir at the University of Rochester, Rochester, NY, USA between 1 March 1996 and 31 December 1996.

The following factors were collected for each patient: age, sex, race, intraocular pressure, visual acuity, extent, location, ${ }^{9}$ laterality, and prior treatment of CMV retinitis, dose of cidofovir, time since diagnosis of CMV retinitis, CD4+ T lymphocyte count, serum creatinine and urine protein concentrations, iris colour, history of diabetes mellitus, and use of concomitant medications at the time of starting cidofovir therapy. We collected only those creatinine and urine protein concentrations measured immediately before infusions, as is standard protocol.

As CD4+ T lymphocyte counts were not consistently obtained before initiation of the cidofovir therapy in all patients, levels obtained within 3 months before commencing cidofovir therapy were used in the study as baseline level. CD4+ T lymphocyte count levels at the time of uveitis also were not available in many patients; thus, any determination within 1 month of onset of uveitis was used. For patients who did not develop uveitis, the highest $\mathrm{CD} 4+\mathrm{T}$ lymphocyte count, serum creatinine, and urine protein level documented during the course of cidofovir therapy were used for comparison with the measurements for the group with uveitis in the data analysis. Data from three patients (one who developed uveitis and two 
Table 1 Comparison of patient baseline characteristics

\begin{tabular}{|c|c|c|c|}
\hline & Patients with uveitis $(n=10)$ & Patients without uveitis $(n=7)$ & $p$ Value \\
\hline Age (mean (SD)) & $38.1(5.5)(n=10)$ & $40.6(4.4)(n=7)$ & 0.34 \\
\hline \multicolumn{4}{|l|}{ Sex } \\
\hline Males & $7(70 \%)$ & $7(100 \%)$ & \multirow[t]{2}{*}{0.11} \\
\hline Females & $3(30 \%)$ & $0(0 \%)$ & \\
\hline \multicolumn{4}{|l|}{ Race } \\
\hline Non-Hispanic white & 4 & 6 & \multirow[t]{4}{*}{0.29} \\
\hline Non-Hispanic black & 4 & 1 & \\
\hline Hispanic & 1 & 0 & \\
\hline Other & 1 & 0 & \\
\hline \multicolumn{4}{|l|}{ Time from CMV retinitis diagnosis to start of cidofovir } \\
\hline therapy (months) & $7.3(5.5)(\mathrm{n}=10)$ & $4.3(6.0)(n=6)$ & 0.33 \\
\hline \multicolumn{4}{|l|}{ Previous anti-CMV retinitis therapy } \\
\hline Ganciclovir & 1 & 1 & \multirow[t]{3}{*}{$0.68^{\star}$} \\
\hline Foscarnet & 1 & 0 & \\
\hline Ganciclovir and foscarnet & 7 & 5 & \\
\hline \multicolumn{4}{|l|}{ Duration of previous anti-CMV retinitis therapy } \\
\hline (months) & $8.0(5.6)(\mathrm{n}=9)$ & $6.2(6.3)(n=10)$ & 0.56 \\
\hline \multicolumn{4}{|l|}{ Medications used during cidofovir treatment } \\
\hline Protease inhibitors & $7(70 \%)$ & $6(86 \%)$ & 0.45 \\
\hline Antiretrovirals & $9(100 \%)$ & $5(100 \%)$ & 1.0 \\
\hline HAART + & $7(70 \%)$ & $4(80 \%)$ & 0.68 \\
\hline Rifabutin & $1(10 \%)$ & $1(14 \%)$ & 0.79 \\
\hline Sulpha antibiotics & $5(56 \%)$ & $4(80 \%)$ & 0.36 \\
\hline Fluconazole & $4(44 \%)$ & $3(60 \%)$ & 0.58 \\
\hline Diabetes mellitus & $0(0 \%)$ & $1(14 \%)$ & 0.22 \\
\hline Number of cidofovir doses $\ddagger$ & $8.0(3.8)(n=10)$ & $6.9(2.5)(n=7)$ & 0.47 \\
\hline CD4+ T lymphocyte count $\left(\times 10^{6} / 1\right)$ & $44.2(75.3)(\mathrm{n}=9)$ & $40.7(54.0)(\mathrm{n}=7)$ & 0.92 \\
\hline \multicolumn{4}{|l|}{ Duration between CD4+ T lymphocyte count } \\
\hline measurement and cidofovir initiation (months) & $1.6(1.4)(\mathrm{n}=10)$ & $1.6(1.6)(\mathrm{n}=7)$ & 0.98 \\
\hline Serum creatinine $(\mathrm{mg} / \mathrm{dl})$ & $1.05(0.29)(\mathrm{n}=10)$ & $1.03(0.32)(\mathrm{n}=7)$ & 0.89 \\
\hline \multicolumn{4}{|l|}{ Proteinuria } \\
\hline $0+$ & 5 & 3 & \multirow[t]{6}{*}{0.77} \\
\hline Trace & 2 & 2 & \\
\hline $1+$ & 3 & 1 & \\
\hline $2+$ & 0 & 0 & \\
\hline $3+$ & 0 & 0 & \\
\hline $4+$ & 0 & 0 & \\
\hline Vision (logMAR units)』 & $0.121(0.178)(n=9)$ & $0.164(0.148)(n=7)$ & 0.62 \\
\hline \multicolumn{4}{|l|}{ Iris colour } \\
\hline Blue & 2 & 2 & \multirow[t]{3}{*}{0.25} \\
\hline Hazel & 1 & 3 & \\
\hline Brown & 6 & 2 & \\
\hline Intraocular pressure $(\mathrm{mm} \mathrm{Hg}) \|$ & $12.6(1.7)(\mathrm{n}=8)$ & $11.7(4.3)(\mathrm{n}=6)$ & 0.62 \\
\hline \multicolumn{4}{|l|}{ CMV retinitis } \\
\hline \multicolumn{4}{|l|}{ Laterality } \\
\hline Right eye & 2 & 2 & \multirow[t]{3}{*}{0.68} \\
\hline Left eye & 5 & 2 & \\
\hline Both eyes & 3 & 3 & \\
\hline \multicolumn{4}{|l|}{ Extent of fundus involved } \\
\hline$<10 \%$ & 2 & 1 & \multirow[t]{4}{*}{0.96} \\
\hline $10-24 \%$ & 3 & 2 & \\
\hline $25-49 \%$ & 2 & 2 & \\
\hline$>50 \%$ & 2 & 1 & \\
\hline $\mathrm{CMV}$ retinitis in zone $1 \#$ & $6(60 \%)$ & $5(71 \%)$ & 0.63 \\
\hline
\end{tabular}

${ }^{\star} \mathrm{p}$ Value is comparison of therapy with foscarnet versus without foscarnet.

†Highly active antiretroviral therapy (the combination of an HIV protease inhibitor with two or more nucleoside analogues). $\neq$ Lesser of number of doses before uveitis or total number of doses.

SSnellen visual acuity was recorded for the right eye in patients without uveitis or with bilateral uveitis, or the affected eye in patients with unilateral uveitis, and converted to logMAR units. ${ }^{11} 12$

$\|$ Intraocular pressure was recorded for the right eye in patients without uveitis or with bilateral uveitis, or the affected eye in patients with unilateral uveitis.

\#Lesions were assigned to the retinal zones described by Holland and associates. ${ }^{9}$

who did not) was censored for statistical analyses involving changes in CD4+ T lymphocyte count levels, as these patients did not have separate measurements before and after initiation of cidofovir therapy.

For quantification of anterior chamber cells, we used measurements recorded in charts; in general, grading of anterior chamber inflammation is done at our institution according to the method of Hogan and associates using a scale of 0 to $4+.^{10}$ For the purposes of this study, an episode of anterior uveitis was defined by one or more of the following: development of symptoms (redness, pain, photophobia) with any degree of anterior chamber cells; an increase of anterior chamber cells by 2 grades or to an absolute level of $\geqslant 3+$; or new inflammatory signs (keratic precipitates or posterior synechiae). The intraocular pressure measurement used for baseline values was obtained from the last eye examination immediately preceding the onset of cidofovir treatment. For intraocular pressure during therapy, we recorded measurements taken at the examination during which uveitis was diagnosed or, for patients without uveitis, the lowest intraocular pressure measured while on cidofovir treatment. Hypotony was defined as a $50 \%$ drop in intraocular pressure from baseline or a pressure of $4 \mathrm{~mm} \mathrm{Hg}$ or less. Corneal oedema was defined as the presence of folds in Descemet's membrane with any degree of loss of corneal transparency. Posterior synechiae were any adhesions present between the posterior iris and the anterior lens capsule.

Visual acuity recorded at each ophthalmological examination was converted into logMAR scores for statistical correlations. 
Table 2 Comparison of patients at the time of uveitis onset compared with maximal levels in patients without uveitis

\begin{tabular}{|c|c|c|c|}
\hline & $\begin{array}{l}\text { Patients with uveitis } \\
(n=10)\end{array}$ & $\begin{array}{l}\text { Patients without } \\
\text { uveitis }(n=7)\end{array}$ & $p$ Value \\
\hline CD4+ T lymphocyte count $\left(\times 10^{6} / 1\right)$ & $103.1(107.1)(n=10)$ & $61.4(57.2)(\mathrm{n}=5)$ & 0.44 \\
\hline \multicolumn{4}{|l|}{ Change in CD $4+\mathrm{T}$ lymphocyte } \\
\hline count from baseline $\left(\times 10^{6} / 1\right)$ & $68.0(76.1)(\mathrm{n}=9)$ & $5.0(10.6)(\mathrm{n}=5)$ & 0.04 \\
\hline Serum creatinine $(\mathrm{mg} / \mathrm{dl})$ & $1.20(0.27)(\mathrm{n}=10)$ & $1.20(0.25)(\mathrm{n}=7)$ & 1.0 \\
\hline \multicolumn{3}{|l|}{ Change in serum creatinine from } & 0.76 \\
\hline \multicolumn{4}{|l|}{ Proteinuria } \\
\hline $0+$ & 1 & 1 & 0.50 \\
\hline Trace & 2 & 0 & \\
\hline $1+$ & 4 & 2 & \\
\hline $2+$ & 3 & 1 & \\
\hline $3+$ & 0 & 1 & \\
\hline $4+$ & 0 & 0 & \\
\hline Vision (logMAR units) ${ }^{\star}$ & $0.362(0.230)(n=10)$ & $0.164(0.148)(\mathrm{n}=7)$ & 0.06 \\
\hline Change in vision (logMAR units) & $0.247(0.195)(\mathrm{n}=9)$ & $0.0(0.0)(\mathrm{n}=7)$ & 0.005 \\
\hline Intraocular pressure $(\mathrm{mm} \mathrm{Hg}) \dagger$ & $7.8(1.9)(\mathrm{n}=9)$ & $9.0(3.8)(\mathrm{n}=5)$ & 0.43 \\
\hline \multicolumn{4}{|l|}{ Change in intraocular pressure from } \\
\hline baseline (mm Hg) & $4.9(2.3)(\mathrm{n}=7)$ & $3.6(2.5)(n=5)$ & 0.39 \\
\hline
\end{tabular}

${ }^{\star}$ Snellen visual acuity was recorded for the right eye in patients without uveitis or with bilateral uveitis, or the affected eye in patients with unilateral uveitis, and converted to logMAR units. ${ }^{11}{ }^{12}$ tIntraocular pressure (IOP) was recorded for the right eye in patients without uveitis or with bilateral uveitis, or the affected eye in patients with unilateral uveitis. For patients without uveitis, the least IOP measurement while on cidofovir therapy was used.

Counting fingers vision was converted to logMAR score of 2.0, hand movements to 3.0, light perception to 4.0 , and no light perception to $5.0 .^{112}$ Visual acuity measured at the last eye examination immediately before the start of cidofovir therapy was recorded as the baseline measurement. For visual acuity during therapy, we recorded measurements at the examination during which uveitis was diagnosed, and for patients without uveitis, the worst visual acuity measured while on cidofovir treatment was used for comparison.

We compared patients who developed uveitis with those who did not to determine whether differences existed between the two populations at baseline. We also compared patient characteristics at the time uveitis developed with the relevant values in patients without uveitis during treatment.

Continuous variables were compared using standard linear analysis of variance and stepwise linear regression analysis. Pre- and post-cidofovir therapy differences were analysed by two tailed paired Student's $t$ test. Categorical variables were compared using the Pearson $\chi^{2}$ test. Kaplan-Meier analysis was

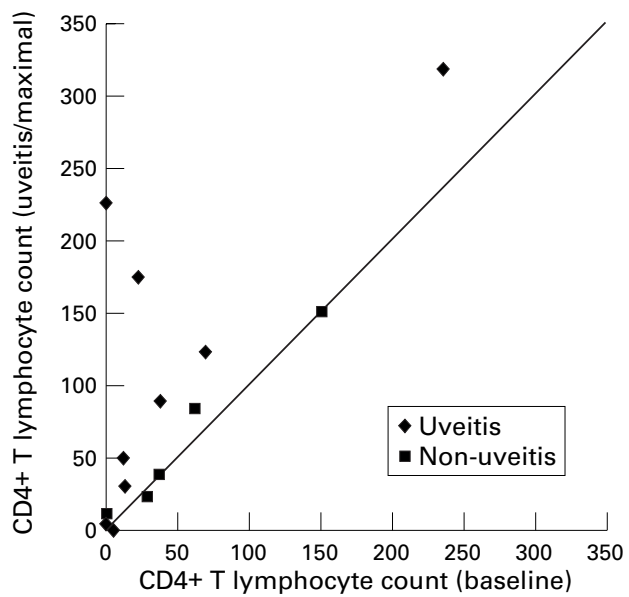

Figure 1 Scatter plot of change in CD4+ T lymphocyte count from baseline to time of uveitis (or maximal level in patients without uveitis). performed to estimate median interval to uveitis. Unless otherwise stated all results are given as mean $(\mathrm{SD})$.

\section{Results}

All patients received cidofovir $5 \mathrm{mg} / \mathrm{kg}$ once weekly for 2 weeks (induction) and then 5 $\mathrm{mg} / \mathrm{kg}$ once every 2 weeks (maintenance). Cidofovir was administered by intravenous infusion in $100 \mathrm{ml}$ of normal saline during a 1 hour period after intravenous hydration with 1 litre of normal saline. All patients received oral probenecid on the day of cidofovir infusion, administered as $2 \mathrm{~g} 3$ hours before each infusion, and $1 \mathrm{~g} 2$ hours and 8 hours after each infusion.

Anterior uveitis occurred in 10 (59\%) of 17 patients. No significant differences between patients who developed uveitis and those who did not were identified at baseline, including in rates of use of protease inhibitors or highly active antiretroviral therapy (Table 1). Patients who developed uveitis had a significantly greater rise in $\mathrm{CD} 4+\mathrm{T}$ lymphocyte count while on cidofovir $\left(68.0(76.1) \times 10^{6} / 1\right)$ than controls $\left(5.0(10.6) \times 10^{6} / 1\right)(\mathrm{p}=0.04)$ (Table 2). Paired analysis revealed that the change in CD4+ T lymphocyte count among patients with uveitis was significant $(p=0.03)$; no significant change occurred in patients without uveitis $(p=0.35)$ (Fig 1$)$. Stepwise linear regression analysis revealed that the increase in CD4+ T lymphocyte count explained 33\% (p $=0.03$ ) of the effect in development of uveitis. The mean CD4+ T lymphocyte count at the time of uveitis $\left(103.1(107.1) \times 10^{6} / 1\right)$ was greater than the maximal CD4+ T lymphocyte count of controls $\left(61.4(57.2) \times 10^{6} / 1\right)$ but was not statistically significant $(\mathrm{p}=0.44)$. Mean follow up time for patients who did not develop uveitis (6.9 (SD 2.5) doses) was not significantly different from mean time to development of uveitis among their counterparts (8.0 (3.8) doses) $(p=0.47)$

Intraocular pressure decline during cidofovir therapy was not significantly different between patients with uveitis, 4.9 (2.3) $\mathrm{mm} \mathrm{Hg}$, and controls, 3.6 (2.5) mm Hg $(\mathrm{p}=0.39)$. Hypotony among patients with uveitis (two $(22 \%)$ of nine) was not significantly more common than among patients without uveitis (one (14\%) of seven) $(p=0.69)$. The decline in logMAR vision among patients with uveitis (0.247 $(0.195))$ was significantly greater than that in controls $(0.0(0.0))(p=0.005)$. As all patients received prophylactic probenecid and intravenous prehydration, the effect of these variables could not be studied.

Table 3 lists the characteristics of the uveitis. Uveitis developed 4.4 (SD 2.5) days (median 3.5) after the preceding infusion of cidofovir, and after 8.0 (3.8) doses (median 8.5) of cidofovir administration. Kaplan-Meier analysis revealed that the median interval to development of uveitis was 11 doses of cidofovir (95\% CI, 5-12 doses). Among patients with unilateral CMV retinitis who developed uveitis, the ipsilateral eye alone developed uveitis in $75 \%$; in the remaining $25 \%$ uveitis was bilateral. In $50 \%$ of patients with bilateral CMV retinitis 
Table 3 Characteristics of uveitis ( $n=10$ cases)

\begin{tabular}{ll}
\hline Anterior chamber cell & \\
$1+$ & $1(10 \%)$ \\
$2+$ & $5(50 \%)$ \\
$3+$ & $2(20 \%)$ \\
$4+$ & $1(10 \%)$ \\
Hypopyon & $2(20 \%)$ \\
Not recorded & $1(10 \%)$ \\
Anterior chamber flare & $2(20 \%)$ \\
$0+$ & $2(20 \%)$ \\
$1+$ & $3(30 \%)$ \\
$2+$ & $1(10 \%)$ \\
$3+$ & $1(10 \%)$ \\
$4+$ & $1(10 \%)$ \\
Not recorded & $2(20 \%)$ \\
Corneal oedema & $6(60 \%)$ \\
Posterior synechiae & $2(22 \%)$ \\
Hypotony & \\
Days since last cidofovir infusion, mean & \\
(median) & $4.4(\mathrm{SD} 2.5)(3.5)$ \\
Number of cidofovir doses before & \\
uveitis, mean (median) & $8.0(\mathrm{SD} 3.8)(8.5)$ \\
Relation to CMV retinitis & \\
Unilateral CMV retinitis (n=8) & \\
$\quad$ Unilateral uveitis, ipsilateral to & \\
$\quad$ CMV retinitis & $6(75 \%)$ \\
$\quad$ Unilateral uveitis, contralateral to & \\
$\quad$ CMV retinitis & $0(0 \%)$ \\
Bilateral uveitis & $2(25 \%)$ \\
Bilateral CMV retinitis (n=2) & $1(50 \%)$ \\
$\quad$ Bnilateral uveitis & $1(50 \%)$ \\
\hline$\quad$
\end{tabular}

who developed uveitis, it was bilateral. All 10 patients reported one or more symptoms (redness, pain, photophobia). Uveitis was accompanied by a significant decrease in logMAR vision (0.247 (0.195) units, $\mathrm{p}=0.004)$. Six $(60 \%)$ patients developed posterior synechiae, two $(20 \%)$ had corneal oedema, and two $(20 \%)$ had a hypopyon. There was no association between the severity of anterior chamber inflammation and CD4+ T lymphocyte count at the time of uveitis $(\mathrm{p}=0.30)$ or change in CD4+ T lymphocyte count $(\mathrm{p}=0.30)$.

Hypotony developed concurrently with uveitis in two $(22 \%)$ patients, one of whom had an intraocular pressure of $4 \mathrm{~mm} \mathrm{Hg}$. There was no significant difference in anterior chamber cell or flare, development of corneal oedema or posterior synechiae, visual acuity, or maintenance dose of cidofovir between patients who developed hypotony and those who did not in the group with uveitis.

Treatment with prednisolone acetate $1 \%$ and cycloplegia effected resolution of inflammation in almost all patients. Topical medications were tapered as the inflammation resolved without recrudescence of uveitis in some patients. The majority of patients, however, had a chronic relapsing course of anterior uveitis often exacerbated by cidofovir infusions. Many patients therefore self medicated with topical corticosteroids, 1 or 2 days before the anticipated arrival of symptoms.

\section{Discussion}

Intravenous cidofovir therapy for CMV retinitis in patients with AIDS was associated with the development of anterior uveitis in 10 (59\%) of 17 patients. Early studies of intravenous cidofovir did not often report uveitis as an adverse reaction. ${ }^{12}$ One reason may be that those cohorts were studied before the widespread availability of HIV protease inhibitors, which promote regeneration of $\mathrm{CD} 4+\mathrm{T}$ lymphocytes, a factor significantly associated with the development of uveitis in our patients.

Our data reveal a significant association between rising CD4+ $\mathrm{T}$ lymphocyte counts and the development of anterior uveitis while on cidofovir therapy. Although CD4+ $\mathrm{T}$ lymphocyte counts were not available for all patients on the first day of cidofovir therapy, the mean duration between CD4+ $\mathrm{T}$ lymphocyte count measurement and cidofovir initiation was not significantly different between patients who developed uveitis and those who did not. While rising CD4+ $\mathrm{T}$ lymphocyte counts were associated with uveitis, there appeared to be no threshold level associated with its occurrence.

In contrast with the results published by Davis et $a l,{ }^{6}$ but in concurrence with Akler et $a l,{ }^{7}$ we did not find the use of protease inhibitors alone to be associated with the development of uveitis. Our data suggest that patients on protease inhibitor therapy are at risk for uveitis if they experience immune restitution, as reflected by elevation in CD4+ T lymphocyte counts. Uveitis itself is not believed to be a complication of protease inhibitor use; the only case report of uveitis associated with protease inhibitor use was in a patient who was also receiving intravenous cidofovir. ${ }^{13}$ Some protease inhibitors have been shown to increase serum concentration of rifabutin, ${ }^{14}$ thereby increasing the risk of uveitis associated with that drug. ${ }^{15} \mathrm{~A}$ similar interaction with cidofovir cannot be excluded by our data.

Our data provide additional support for the association between intravenous cidofovir therapy and anterior uveitis. Uveitis was observed only after multiple injections of cidofovir, suggesting that accumulation of the drug is necessary to initiate the inflammatory reaction.

A few anti-infective agents have been associated with uveitis. Rifabutin prophylaxis and therapy of Mycobacterium avium complex has, in a dose related manner, been linked to a fulminant hypopyon uveitis with inflammatory vitreous opacities that resolves with corticosteroid therapy. ${ }^{16-18}$ Rifabutin use was not significantly associated with uveitis in our patients. The incidence of uveitis among rifabutin users in our cohort is much higher than the reported incidence of rifabutin associated uveitis, ${ }^{18}$ although our sample size is small. None of our patients had inflammatory vitreous opacities, and of the two with hypopyon one had a history of rifabutin use at the time of starting cidofovir therapy but not at the time of uveitis.

The cause of cidofovir associated uveitis remains unclear. One possibility is direct toxicity, but this must be reconciled with the fact that uveitis resolves despite continuation of the drug. The concomitant use of probenecid decreases the incidence of uveitis associated with intravitreal cidofovir. ${ }^{8}$ Probenecid inhibits renal tubular secretion of cidofovir ${ }^{19}$ and may inhibit secretion from the ciliary body, which shares many of the transport mechanisms in the kidney, resulting in decreased intraocular 
drug levels. Hypotony associated with intravitreal cidofovir appears to be due to damage to the ciliary body. ${ }^{20}{ }^{21}$ Akler et $a l^{7}$ found an association between increasing serum creatinine while receiving cidofovir and development of uveitis, suggesting that decreased renal excretion may lead to toxic accumulation of drug. We could not confirm this result.

Cidofovir associated uveitis is quite different in its manifestation from immune recovery uveitis (IRU), which also is associated with rising CD4+ T lymphocyte counts. IRU is a distinctive type of vitreous inflammation, sometimes associated with optic disc oedema, macular oedema, epiretinal membrane formation, or cataract. ${ }^{22-26}$ Essentially it is a posterior or intermediate uveitis and is not associated with posterior synechiae or granulomatous keratic precipitates, which frequently are observed in cidofovir associated uveitis. CMV infection itself can be associated with a low grade anterior chamber reaction but has not been reported to result in the type of marked inflammation observed in our patients with uveitis.

We found that patients with uveitis tended to have the diagnosis of CMV retinitis for a longer period of time (7.3 (SD 5.5) months) than those without uveitis (4.3 (6.0) months) $(\mathrm{p}=0.33)$. This trend was not found to be statistically significant because of insufficient power $(0.15)$ owing to the small sample sizes. However, this difference may be clinically meaningful as breakdown of the blood-ocular barrier is significantly higher in eyes with CMV retinitis than those without and increases with duration of CMV retinitis ${ }^{27}$ and may promote higher intraocular cidofovir levels. Patients with CMV retinitis in zone $1,{ }^{9}$ compared with those with peripheral disease, have a greater degree of blood-ocular barrier breakdown as measured by laser flare photometry. ${ }^{27}$ However, we found no association between uveitis and zone 1 involvement. Davis et $a l^{6}$ reported an association between diabetes mellitus, in which the blood-ocular barrier is altered, and the development of uveitis. We, like Akler et $a l,{ }^{7}$ were unable to confirm this association as only one of our patients had diabetes mellitus and he did not develop uveitis.

We found that patients who developed uveitis had a greater rise in CD4+ T lymphocyte counts while on cidofovir, and there is much evidence linking CD4+ T lymphocytes to the pathogenesis of uveitis. Experimental autoimmune uveitis induced by retinal $S$ antigen ${ }^{28}$ or by melanin associated protein ${ }^{29}$ is mediated by CD4+ T lymphocytes. CD4+ T lymphocytes are found in high levels in the aqueous humour $^{30}$ and retina ${ }^{31}$ of patients with uveitis. The immunopathogenesis of these entities resembles that of recurrent iridocyclitis in humans ${ }^{32}$ and antibodies against CD 4+ T lymphocytes inhibit their development. ${ }^{33}$

The development of uveitis in these patients is important because uveitis in an immunocompromised patient can be ominous and warrant extensive diagnostic evaluation and trigger potentially morbid diagnostic proce- dures or systemic treatment. Recognition of cidofovir associated uveitis can save the patient potentially invasive interventions and lead to proper management.

It has been suggested that the cidofovir associated hypotony is an accompaniment of severe drug associated uveitis. ${ }^{6}$ However, our data suggest that hypotony and uveitis are independent complications of cidofovir use as one patient without uveitis developed hypotony and seven patients with uveitis never developed hypotony. In addition, probenecid decreases the incidence of uveitis but does not alter intraocular pressure in eyes treated with intravitreal cidofovir. ${ }^{20}$ Patients with hypotony and uveitis may simply be manifesting concurrent complications. Alternatively, although we did not find a relation between uveitis and intraocular pressure, it is possible that hypotony may complicate severe uveitis.

While the development of hypotony warrants discontinuation of the drug, uveitis alone may not preclude continuing cidofovir as it responds to topical corticosteroids and cycloplegics. Although manageable, cidofovir associated uveitis can be accompanied by pain, visual loss, posterior synechiae, and hypotony, and may be a consideration in selecting among treatments for CMV retinitis. These patients may benefit from ganciclovir or foscarnet therapy as an alternative to cidofovir; in some cases, specific anti-CMV medications may be discontinued in patients experiencing a sustained CD4+ $\mathrm{T}$ lymphocyte count rise above 100 cells $\times 10^{6} / 1$ on HAART for at least 3 months. ${ }^{34} 35$

Supported, in part, by the Heed Ophthalmic Foundation, AOSKnapp Testimonial Fund, and a Foundation Fighting Blindness Career Development Award (to Dr Ambati), and an unrestricted grant from Research to Prevent Blindness (to University of Rochester)

1 Lalezari JP, Stagg RJ, Kuppermann BD, et al. Intravenous cidofovir for peripheral cytomegalovirus retinitis in patients with AIDS. A randomized, controlled trial. Ann Intern Med 1997;126:257-63.

2 Studies of Ocular Complications of AIDS Research Group. Parenteral cidofovir for cytomegalovirus retinitis in patients with AIDS: The HPMPC peripheral cytomegalovirus retinitis trial. A randomized, controlled trial. Ann Intern Med 1997;126:264-74.

3 Kirsch LS, Arevalo JF, de la Paz EC, et al. Intravitreal cidofovir (HPMPC) treatment of cytomegalovirus retinitis in patients with acquired immune deficiency syndrome. Ophthalmology 1995;102:533-43.

4 Rahhal FM, Arevalo JF, Chavez de la Paz E, et al. Treatment of cytomegalovirus retinitis with intravitreous cidofovir in patients with AIDS. Ann Intern Med 1996;125:98-103.

5 Rahhal FM, Arevalo JF, Munguia D, et al. Intravitreal cidofovir for the maintenance treatment of cytomegalovirus retinitis. Ophthalmology 1996;103:999-1000.

6 Davis JL, Taskintuna I, Freeman WR, et al. Iritis and hypotony after treatment with intravenous cidofovir for cytometony after treatment with intravenous cidofovir for cyton
galovirus retinitis. Arch Ophthalmol 1997;115:733-7.

7 Akler ME, Johnson DW, Burman WJ, et al. Anterior uveitis and hypopyon after intravenous cidofovir for the treatment of cytomegalovirus retinitis. Ophthalmology 1998;105:651-7.

8 Chavez de la Paz E, Arevalo JF, Kirsch LS, et al. Anterior nongranulomatous uveitis after intravitreal HPMPC (cidofovir) for the treatment of peripheral cytomegalovirus retinitis. Ophthalmology 1997;104:539-44.

9 Holland GN, Buhles WC, Mastre B, et al. A controlled retrospective study of ganciclovir treatment for cytomegalovirus retinopathy: use of a standardized system for the assessment of disease outcome. Arch Ophthalmol 1989;107:175966.

10 Hogan MH, Kimura SJ, Thygeson P. Signs and symptoms of uveitis: I. Anterior uveitis. Am f Ophthalmol 1959;47: 155-70.

11 Westheimer G. Scaling of visual acuity measurements. Arch Ophthalmol 1979;97:327-30.

12 Holladay JT, Prager TC. Mean visual acuity. Am f Ophthalmol 1991;111:372-4. 
13 Gariano RF, Cooney EL. Uveitis following administration of the protease inhibitor indinavir in a patients with AIDS. of the protease inhibitor ind
Clin Infect Dis 1997;24:529.

14 Piscitelli SC, Flexner C, Miner JR, et al. Drug interactions in patients infected with human immunodeficiency virus. Clin Infect Dis 1996;23:685-93.

15 Sun E, Heath-Chiozzi M, Cameron DW, et al. Concurrent ritonavir and rifabutin increase risk of rifabutin-associated adverse event. Abstract No B171. XI International Conference on AIDS, Vancouver, Canada, 8 July 1996.

16 Jacobs DS, Piliero PJ, Kuperwaser MG, et al. Acute uveitis associated with rifabutin in patients with human immunodeficiency virus infection. Am f Ophthalmol 1994;118:805-6.

17 Chaknis MJ, Brooks SE, Mitchell KT, et al. Inflammatory opacities of the vitreous in rifabutin-associated uveitis. $A m$ f Ophthalmol 1996;122:580-2.

18 Shafran SD, Singer J, Zarowny DP, et al. A comparison of two regimens for the treatment of Mycobacterium avium complex bacteremia in AIDS: rifabutin, ethambutol, and clarithromycin versus rifampin, ethambutol, clofazimine, clarithromycin versus rifampin, ethambutol, clofazimine, and ciprofloxacin: Canadian HIV Trials Network

19 Cundy KC, Li ZH, Lee WA. Effect of probenecid on the distribution, metabolism, and excretion of cidofovir in rabbits. Drug Metab Dispos 1996;24:315-21.

20 Banker AS, Arevalo JF, Munguia D, et al. Intraocular pressure and aqueous humor dynamics in patients with AIDS treated with intravitreal cidofovir AIDS treated with intravitreal cidofovir (HPMPC) for cytomegalo $168-80$.

21 Taskintuna I, Banker AS, Rao, NA, et al. An animal model for cidofovir (HPMPC) toxicity:intraocular pressure and histopathologic effects. Exp Eye Res 1997;64:795-806.

22 Zegans ME, Walton RC, Holland GN, et al. Transient vitreous inflammatory reactions associated with combination antiretroviral therapy in patients with AIDS and cytomegalovirus retinitis. Am ₹ Ophthalmol 1998;125:292-300.

23 Karavellas MP, Lowder CY, Macdonald C, et al. Immune recovery vitritis associated with inactive cytomegalovirus retinitis: a new syndrome. Arch Ophthalmol 1998;116: 169-75.
24 Herbort CP, Chave JP. Cicatrization of cytomegalovirus retinitis following introduction of highly active antiretroviral therapy:uveitis as a possible indicator of good ocular prognosis.

25 Henderson HW, Mitchell SM. Treatment of immune recovery vitritis with local steroids. Br F Ophthalmol 1999;83: 540-5.

26 Cassoux N, Lumbroso L, Bodaghi B, et al. Cystoid macular oedema and cytomegalovirus retinitis in patients with HIV disease treated with highly active antiretroviral therapy. $\mathrm{Br}$ f Ophthalmol 1999;83:47-9.

27 Magone MT, Nussenblatt RB, Whitcup SM. Elevation of aser flare photometry in patients with cytomegalovirus retinitis and AIDS. Am f Ophthalmol 1997;124:190-8.

28 Nussenblatt RB. Proctor lecture. Experimental autoimmune uveitis: mechanisms of disease and clinical therapeutic indications. Invest Ophthalmol Vis Sci 1991;32:3131-41.

29 Kim MC, Kabeer NH, Tandhasetti MT, et al. Immunohistochemical studies on melanin associated antigen induced experimental autoimmune anterior uveitis. Curr Eye Res 1995;14:703-10.

30 Chan CC, Hikita N, Dastgheib K, et al. Experimental melanin-protein-induced uveitis in the Lewis rat. Immun pathologic processes. Ophthalmology 1994;101:1275-80.

31 Ohta K, Norose K, Wang XC, et al. Apoptosis-related fas antigen on memory $\mathrm{T}$ cells in aqueous humor of uveitis patients. Curr Eye Res 1996;15:299-306.

32 Fujikawa LS, Haugen JP. Immunopathology of vitreous and retinochoroidal biopsy in posterior uveitis. Ophthalmology 1990;97:1644-53.

33 Atalla L, Linker-Israeli M, Steinman L, et al. Inhibition of autoimmune uveitis by anti-CD4 antibody. Invest Ophthalmol Vis Sci 1990;31:1264-70.

34 Vrabec TR, Baldassano VF, Whitcup SM. Discontinuation of maintenance therapy in patients with quiescent cytomegalovirus retinitis and elevated CD4+ counts. Ophthalmology 1998;105:1259-64.

35 Macdonald JC, Torriani FJ, Morse LS, et al. Lack of reactivation of cytomegalovirus (CMV) retinitis after stopping tained elevations in CD4 T cells in response to highly active antiretroviral therapy. $\mathcal{F}$ Infect Dis 1998;188:1182-7. 